\title{
ON THE CONVERGENCE CHARACTERISTICS OF THE MODAL ANALYSIS OF FUSED FIBER-OPTIC COUPLERS
}

\author{
Yao-Chun Tsai, Bo-Rui Jiang*, and Hung-chun Chang** \\ Graduate Institute of Communication Engineering, National Taiwan University \\ Taipei, Taiwan 106-17, R.O.C. \\ Phone: +886-2-23635251 ext. 513, Fax: +886-2-23638247 \\ E-mail: hcchang@cc.ee.ntu.edu.tw \\ * Graduate Institute of Electro-Optical Engineering, National Taiwan University \\ **Also with the Department of Electrical Engineering and the Graduate Institute of Electro-Optical Engineering, \\ National Taiwan University
}

Abstract-Analysis of the birefringent property of the fused fiber-optic coupler needs very accurate calculation of modal propagation constants. We study the numerical convergence of such calculation using the boundary integral method for both $2 \times 2$ and $3 \times 3$ couplers.

This study concerns the accurate analysis of the birefringent property of the fused fiber-optic coupler. Such coupler is made by the fusing and tapering technology [1] and, other than the usual function of optical power splitting, it can be designed to work as a polarization beamsplitter (PBS) [2]. PBSs based on extremely weakly fused structures have been fabricated [3] and numerical modeling of their characteristics has been performed [4], [5]. The cross-sectional structure in the coupling region has dumbbell shape, which becomes close to the touching circular shape for the extremely weakly fused condition, and it is a strongly guiding composite waveguide since the region external to the reduced fiber cladding is air in our case. Practically, only the lowest-order even and odd modes are excited. The coupling coefficient which is defined as half the difference between the even and odd supermode propagation constants depends on the polarization state. The difference in the coupling coefficient between orthogonal polarization states is called form birefringence. It is the form birefringence that causes the polarization splitting in the coupler. We have demonstrated that the form birefringence is small, and accurate calculation of such small quantity is crucial in the modeling [4]. The surface integral equation method (SIEM) [6] which is a boundary integral method is a suitable technique for solving the modes on the coupler. We have employed the method to calculate the vectorial modes and polarizationdependent coupling coefficients and the corresponding form birefringence. We have previously shown that good convergence of the form birefringence calculation requires dividing the contour of the waveguide boundary into a large number of segments, and have developed a nonuniform scheme for dividing the contour to save computing time and memory [4]. In this paper we examine in more detail the convergence of the form birefringence calculation by considering finer uniform division along the waveguide boundary and extend the analysis to the case of collinear $3 \times 3$ coupler structure. We also examine the boundary fields near the touching point of adjacent fibers, which are found to change rapidly and require sufficiently fine boundary division to reach convergent calculated profiles.

Acknowledgments-This work was supported by the National Science Council of the Republic of China under Grant NSC90-2215-E-002-024.

\section{REFERENCES}

[1] B. S. Kawasaki, K. O. Hill, and R. G. Lamont, "Biconical-taper single-mode fiber coupler," Opt. Lett., 6, 327-328 (1981).

[2] M. S. Yataki, D. N. Payne, and M. P. Varnham, “All-fiber polarizing beamsplitter," Electron. Lett., 21, 249251 (1985).

[3] C. W. Wu, T. L. Wu, and H. C. Chang, "A novel fabrication method for all-fiber, weakly fused, polarization beamsplitters," IEEE Photon. Technol. Lett., 7, 786-788 (1995).

[4] S. W. Yang and H. C. Chang," "Numerical modeling of weakly fused fiber-optic polarization beamsplitters - Part I: Accurate calculation of coupling coefficients and form birefringence," J. Lightwave Technol., 16, 685-690 (1998).

[5] S. W. Yang, T. L. Wu, C. W. Wu, and H. C. Chang," "Numerical modeling of weakly fused fiber-optic polarization beamsplitters-Part II: The three-dimensional electromagnetic model," J. Lightwave Technol., 16, 691-696 (1998).

[6] C.-C. Su, . "A surface integral equations method for homogeneous optical fibers and coupled image lines of arbitrary cross sections," IEEE Trans. Microwave Theory Tech., MTT-33, 1114-1120 (1985). 